\section{Failure of Spinal Anesthesia with Marcaine (Bupivacaine): Recent Cluster of Cases}

Spinal anesthesia has been used for over a century and has gained popularity, particularly in the past few decades, as an excellent approach in obstetrics and certain postoperative situations. The published failure rate for spinal anesthesia is less than $3 \%,{ }^{1}$ but in that report, failure was defined as "failure to develop an adequate block despite successful dural puncture", and the cases would thus have included partial or incomplete block. ${ }^{1}$ The incidence of complete failure of spinal block (with no sensory or motor block), despite cerebrospinal fluid flow, is substantially lower. A variety of factors have been implicated in failure of spinal block, including movement of the needle during injection, large intrathecal volume, technical errors, miscalculation of the dose, maldistribution of the local anesthetic, presence of extradural cysts, and anatomic sacral restriction. ${ }^{2,3}$

In June and July 2008, a cluster of 3 cases of complete failure of spinal block after spinal administration of $0.75 \%$ Marcaine (bupivacaine; lot 64095DD, expiry April 1, 2011 [20110401]) occurred at our site. The cases were reported to the manufacturer, Hospira Healthcare Corporation. In these 3 cases, the anesthesia was performed by 2 different experienced anesthesiologists using a standard technique (aseptic technique, infiltration with lidocaine $1 \%$, aspiration of cerebrospinal fluid at the beginning and end of injection). My colleagues and I are aware of 5 similar cases of partially or completely ineffective spinal block with the same drug at 2 other area hospitals within the past few months (lot 67120DD, expiry July 1, 2011 [20110701]). It appears to us that there has been a recent increase in the incidence of spinal-block failure. We have reported one of these recent cases of complete failure, which occurred at our affiliated hospital, to Hospira and to the Canada Vigilance Regional Office of Health Canada. Hospira has stated that the reported lots (64095DD and 67120DD) met all manufacturing specifications.

Clusters of inadequate spinal anesthesia have previously been reported in the literature. In one of the largest clusters, reported by Smiley and Redai, ${ }^{4} 11$ cases of either inadequate or completely failed spinal block despite cerebrospinal fluid flow occurred in a 7-month period. Our recent cluster of cases of complete failure of spinal anesthesia, as well as similar experiences at other institutions, is of great concern. Centres are encouraged to report all cases of failed spinal block with spinal Marcaine to the manufacturer (Hospira, 1-866-488-6088; choose option 7) and to Health Canada (1-866-234-2345 or online at http://www.healthcanada.gc.ca/medeffect).

In addition, my colleagues and I would be interested to hear from anyone who has had a similar experience recently and from anyone who can provide a possible explanation.

References

1. Juhani TP, Hannele H. Complications during spinal anesthesia for cesarean delivery: a clinical report of one year's experience. Reg Anesth 1993;18(2):128-131

2. Spiegel JE, Hess P. Large intrathecal volume: a case of true failed spinal anesthesia. J Anesthesia 2007;21(3):399-402.

3. Hoppe J, Popham P. Complete failure of spinal anaesthesia in obstetrics. Int J Obstet Anesth 2007;16(3):250-255.

4. Smiley R, Redai I. More failed spinal anesthetics with hyperbaric bupivacaine. Int J Obstet Anesth 2004;13(2):131-134.

Marianne J Nagata, BSCPhm, ACPR, RPh

Acute Pain Service

Department of Surgery

William Osler Health Centre

Etobicoke General Hospital

Toronto, Ontario

e-mail: Marianne_nagata@oslerhc.org 